\title{
ADMINISTRACIÓN ESTRATÉGICA DEL SERVICE-DOMINANT LOGIC EN EL NEURODISEÑO DE UN SERVICIO DE GESTIÓN Y CARTAS VIRTUALES DE RESTAURANTES ${ }^{*}$ \begin{abstract}
STRATEGIC ADMINISTRATION OF THE SERVICE-DOMINANT
\end{abstract} LOGIC IN THE NEURODESIGN OF A MANAGEMENT SERVICE AND VIRTUAL LETTERS OF RESTAURANTS
}

Recibido: 23 de octubre de 2020

Evaluado: 21 de noviembre de 2020

Aprobado: 16 de diciembre de 2020

\author{
Daniel Puente Berdasco ${ }^{*}$ \\ Universidad Politécnica de Valencia \\ Orcid: https://orcid.org/0000-0003-2322-4018 \\ Carlos Pinilla Díaz ${ }^{* \star *}$ \\ Corporación Universitaria Minuto de Dios - UNIMINUTO \\ Orcid: https://orcid.org/0000-0002-1833-9366 \\ Juan Felipe Guzmán ${ }^{\star \star \star \star}$ \\ Universidad Metropolitana de Educación, Ciencia y Tecnología \\ Orcid: https://orcid.org/0000-0002-2161-6344
}

Cómo citar este artículo: Puente Berdasco, D., Pinilla Díaz, C., y Guzmán, J.F. (2021). ADMINISTRACIÓN ESTRATÉGICA DEL SERVICEDOMINANT LOGIC ENEL NEURODISEÑO DE UN SERVICIO DE GESTIÓN Y CARTAS VIRTUALES DE RESTAURANTES. Revista Estrategia Organizacional, 10(1), https://doi.org/10.22490/25392786.4550

\footnotetext{
* $\quad$ Artículo de investigación.

** Ingeniero Industrial en Mecánica, Máster en Gestión del Diseño Industrial, Máster en Ingeniería del Diseño, Phd (c) Director Neuro Service Design. S.L, Docente Universidad EAN. Correo electrónico: dapueber@etsid.upv.es

*** Ingeniero de Sistemas y Especialista en Gerencia de la Calidad de la Universidad del Magdalena, Máster en Administración y Dirección de Empresas, Docente Corporación Universitaria de Dios CT Pitalito. Correo electrónico: carlos.pinilla-d@uniminuto.edu.co.

*** Magister en Administración y planificación, Especialista en Pedagogía para el Desarrollo dela aprendizaje Autónomo, Administrador de empresas y Psicólogo. Docente del programa de Administración de empresas. de la Universidad Nacional Abierta y a Distancia.
} 


\title{
RESUMEN
}

Introducción: Avanzar en materia de diseño de servicios se ha convertido en una necesidad imperante para las empresas que buscan ofrecer a sus clientes opciones cada vez más innovadoras que los vincule emocionalmente a sus propuestas y los fidelice. Metodología: Esta investigación tiene como objetivo identificar la importancia de utilizar los avances científicos y tecnológicos en el diseño de servicios, enfatizando en la conexión emocional con el usuario que proveen filosofías de diseño como la ingeniería Kansei y el design thinking. Resultados: Con la aplicación práctica, basada en el uso del eye-tracking y los diferenciales semánticos, se logra evidenciar que los avances tecnológicos, adecuadamente enfocados al diseño de servicio, facilitan el conocimiento del cliente y por ende el desarrollo de soluciones más ajustadas a sus necesidades.

Palabras clave: diseño de servicios, ingeniería Kansei, diferencial semántico, eye-tracking, design thinking, administración estratégica.

\begin{abstract}
Introduction: Advancing in the design of services has become an imperative need for companies that seek to offer their customers increasingly innovative options that emotionally link them to their proposals and retain them. Methodology: This research aims to identify the importance of using scientific and technological advances in the design of services, emphasizing the emotional connection with the user provided by design philosophies such as Kansei engineering and design thinking. Results: With the practical application, based on the use of eye-tracking and semantic differentials, it is possible to show that technological advances, adequately focused on service design, facilitate customer knowledge and therefore the development of solutions more adjusted to your needs.
\end{abstract}

Keywords: service design, Kansei engineering, semantic differential, eye-tracking, design thinking, strategic management. 


\section{INTRODUCCIÓN}

La economía se centró, en primer lugar, en la distribución e intercambio de productos básicos y manufacturados (Marshall, 1927; Shaw, 1912\& Smith, 1904), que, durante la Segunda Guerra Mundial, impulsó el crecimiento de la industrialización en los países desarrollados y pasa a considerarse el nuevo rol que desempeñan los servicios en las economías globales (Galindo Martin, 2009). Aunado a esto, a partir de los años cincuenta, se consolida un enfoque de servicio orientado a satisfacer las necesidades del cliente (Drucker, 1954; Levitt, 1960 \& McKitterick, 1957).

En los últimos años el mercado se ha desplazado de una lógica basada en bienes tangibles, hacia el intercambio de bienes intangibles, habilidades, conocimientos y procesos; lográndose una lógica dominante más amplia e inclusiva, que integra los bienes con los servicios. Se ha trascendido de una visión goods-dominant logic, en donde la producción tangible y las transacciones eran partes centrales del proceso comercial, a una visión service-dominant logic, donde el foco comercial se basa en lo intangible, los procesos de intercambio y las relaciones con los stakeholders (Vargo \& Lush, 2004). Según el Fondo Monetario Mundial (2012), los servicios concentraban más de las dos terceras partes del empleo total y un $63,3 \%$ del producto interno bruto mundial.

Como explica Gummesson (1995, pp. 250-51): “Los consumidores no compran bienes o servicios. La tradicional división entre bienes y servicios ha quedado desfasada. No es una cuestión de redefinir los servicios desde el punto de vista de los consumidores; las actividades acarrean servicios, las cosas acarrean servicios. El relevo en el enfoque hacia los servicios es un relevo desde la perspectiva de los medios y el productor a la perspectiva de la utilización del consumidor". Demostrando así, que un producto adquiere valor añadido según el factor de intangibilidad apreciado por el consumidor frente a las materias primas que lo componen.

La relación cliente-empresa ha cambiado, pero también la ciencia, el conocimiento y las relaciones sociales. Atrás quedaron los tiempos en los que las compañías indicaban a los consumidores lo que debían comprar y por qué y éstos le creían. Por lo tanto, es necesaria una estrategia que ayude a vender en tiempos difíciles (Ruiz, 2013). 
El objetivo de esta investigación se centra en establecer una base teórica y metodológica que permita conocer, conceptual y empíricamente, la importancia de utilizar los avances tecnológicos y científicos en la planificación y diseño de servicios, enfatizando en la aplicación de estas, desde una perspectiva emocional, y su relación con filosofías de diseño como la ingeniería Kansei y el design thinking.

Esta investigación se compone de cinco secciones. En la sección 2 sepresenta la fundamentación teórica de los servicios y su diseño desde una perspectiva emocional. La sección 3 aborda la metodología utilizada para la recolección de datos y su respectivo análisis. En la sección 4 se desarrolla el proyecto que incluye la planificación, diseño y aplicación de las pruebas con el eyetracking y la encuesta de percepción. En la sección 5 se relacionan los resultados obtenidos de la interpretación de los diferenciales semánticos, la encuesta y los datos del seguimiento ocular. Finalmente, se exponen las conclusiones desde una perspectiva teórica y experimental.

\section{DEFINICIÓN DE SERVICIO}

La OEDC (2000) define los servicios como un grupo diverso de actividades económicas que incluyen a los subsectores de alta tecnología y aquellos intensivos en conocimiento, mano de obra y trabajo de baja calificación. En varios aspectos, los servicios se diferencian de la manufactura, pero estas distinciones hoy en día se están disipando, especialmente porque los servicios están incorporando valor añadido procedente de las personas en forma de trabajo, asesoramiento, habilidades de gestión, formación e intermediación.

Asimismo, Hunter (2015), director de diseño del Design Council de Inglaterra, define servicio como lo que se usa personalmente, pero de lo que no se es propietario. A partir de estos conceptos, Aurich et al. (2010) identifica cuatro características de los servicios:

1. Intangibilidad: Enmarca la esencia del servicio y representa ideas y conceptos que no pueden percibirse de forma directa a través de los sentidos.

2. Separabilidad: Son producidos, vendidos y consumidos simultáneamente. 
3. Heterogeneidad: No se pueden estandarizar, desde la perspectiva del cliente existe una gran variación en los servicios en función de su percepción.

4. Caducidad: Los servicios no se pueden almacenar, y al ser fluctuante su demanda, puede generar pérdidas para el proveedor.

Hara (2006) asegura que un servicio tiene como objetivo cambiar el estado del ente receptor, a partir de una actividad o evento realizado por un proveedor. Así, el usuario solo se encontrará satisfecho en la media en que su estado cambie a un estado nuevo y deseado.

Mayer et al. (2003) indica que el proceso de servicio considera dos elementos principales (ver Figura 1):

- Montaje del servicio (Process of Services Assembly - PSA), como elemento técnico.

- Entrega del servicio (Process of Services Delivery - PSD), como elemento funcional.

A estos procesos se le definen algunos filtros de percepción del consumidor (CPPS), cuyo objetivo consiste en evaluar y modificar la percepción de los elementos del servicio, generando satisfacción o insatisfacción.

Albretch (1992) realiza una construcción básica del servicio situando "momentos de verdad" que son controlados por cada empleado y/o sistema que interactúa con el cliente y que representa eventos en los que el cliente obtiene una percepción de calidad sobre el servicio. Este ciclo del servicio es una herramienta que facilita a las organizaciones los procesos de asistencia a sus clientes

\section{DE LA LÓGICA DOMINANTE BUENA A LA LÓGICA DOMINANTE DEL SERVICIO}

Vargo y Lusch (2004) establecen un nuevo marco teórico donde se hace transición de una economía basada en bienes a una basada en servicios, logrando que lo tangible se complemente con lo intangible. 
Una parte importante de este proceso económico es la utilización de recursos que a través de diferentes medios y transformaciones se les otorga un valor para alcanzar una venta competitiva. Malthus (1798) establece que una población en constante crecimiento prontamente agotaría sus recursos, entendiendo estos recursos como elementos naturales. Los recursos se han entendido como "objetos" estáticos que se adquieren para obtener una ventaja competitiva, pero en los últimos 50 años los recursos no solo son materiales, sino que se entienden también como intangibles y dinámicos, como lo es, por ejemplo, el conocimiento.

Dado este cambio de perspectiva sobre los recursos, Constantin y Lusch (1994) han establecido la siguiente clasificación:

- Operand resources: Son principalmente recursos físicos como bienes o materias primas que sometidas a un proceso de transformación generan un producto más elaborado. Como estos recursos eran y son finitos, quien disponía de ellos se consideraba rico. Asimismo, las empresas aumentaban su valor en función de su capacidad para convertir esos recursos en productos terminados y lanzarlos al mercado a un bajo coste.

- Operant resources: Se basan en conocimientos y habilidades que producen un efecto por sí mismos. Estos recursos generalmente permiten aumentar el valor de los recursos tangibles.

Desde la perspectiva de la service-dominant logic se percibe a los operant resources su parte primaria. Este cambio en la primacía de los recursos tiene implicaciones en el intercambio de procesos, en la dinámica del mercado y en como los clientes son percibidos por las organizaciones.

En función de lo anterior, Vargo y Lusch (2004 y modificaciones en 2008) establecen unas premisas fundacionales del service-dominant logic (ver Tabla 1): 
Tabla 1. Premisas fundacionales de la service-dominant logic.

\begin{tabular}{|l|l|}
\hline \multicolumn{1}{|c|}{ Premisas Fundacionales } & \multicolumn{1}{c|}{ Service-Dominant Logic } \\
\hline PF1 & El servicio es la unidad fundamental de intercambio. \\
\hline PF2 & El intercambio indirecto enmascara la unidad principal de intercambio. \\
\hline PF3 & Los bienes son meros mecanismos para proveer servicio. \\
\hline PF4 & El conocimiento es la principal fuente de ventaja competitiva. \\
\hline PF5 & Todas las economías son economías de servicio. \\
\hline PF6 & El cliente siempre es coproductor. \\
\hline PF7 & La empresa solo puede hacer proposiciones de valor. \\
\hline PF8 & Un enfoque centrado en el servicio está orientado al cliente y es relacional. \\
\hline PF9 & Todos los agentes sociales son integradores de recursos. \\
\hline P10 & El valor es únicamente determinado por el usuario. \\
\hline
\end{tabular}

Fuente: Vargo y Lusch (2004 y modificaciones en 2008)

Esta serie de premisas puede resumirse en los cuatro siguientes axiomas:

1. Los recursos deben aplicarse en un intercambio recíproco de servicio.

2. Deben integrarse recursos intangibles y dinámicos que creen nuevos recursos.

3. Se consigue co-creación de valor mediante interacciones y colaboraciones de actores.

4. La importancia o medida del valor estará condicionada por el contexto en el que fue creado.

Esta visión del service-dominant logic es un cambio completo de paradigma desde la perspectiva de las relaciones económicas y de consumo. Pasando de un estado en el que la empresa lanza un producto al mercado añadiéndole valor, a entrar en un sistema de relaciones en el que los diferentes agentes del proceso, y principalmente el cliente, juegan un papel importante como parte activa en la creación de valor de los servicios, adaptando de esta forma el producto a las necesidades reales del usuario. Así, se promueve una relación más humanística del proceso, logrando que la comunicación entre las partes deje en segundo plano la producción y publicidad. 
Con este enfoque, los bienes conforman la parte tangible del intercambio en vez de ser un fin en sí mismos. Si la empresa se centra demasiado en el producto, puede perder la visión de utilidad que recibe el usuario a través del servicio, siendo esto último lo realmente importante.

Esta tendencia hacia los servicios se ha ido apoderando de la industria manufacturera a través de la implementación del proceso de servitización. Este proceso consiste en añadir o integrar servicios a la oferta de productos como una estrategia para logar una mejora de su posición competitiva (Vandermerwe y Rada, 1988; Baines y Lightfoot, 2013).

Nelly (2013) lo define como un proceso de transformación que tiene lugar en las empresas que deciden desarrollar determinadas capacidades que estiman necesarias para proveer o facilitar servicios y soluciones suplementarias a sus ofertas tradicionales de productos. Desde otra perspectiva, Kamp, Ochoa y Díaz (2016) interpretan la servitización como una manera distinta de concebir relaciones entre proveedores de activos y sus usuarios.

Actualmente, según Iriarte (2016), son muchas las organizaciones servitizadas que ofrecen servicios avanzados con base en contratos de disponibilidad garantizada (IBM, Hilti, Machinery Link, ABB Turbo Systems Otis), contratos basados en la actividad (Rolls-Royce, BASF Coatings, Xerox, Smartville) o en soluciones llave en mano (Heidelberg Printing Machines, Lantal Textiles, Tetra Pak, 3M). Pero a pesar de estos avances, se ha detectado que la servitización no considera, en su conjunto, la experiencia ofertada por el cliente, lo que implica el desarrollo de estrategias basadas en el diseño de servicios (Bitner y Brown, 2008).

Finalmente, Iriarte (2016) concluye que el diseño de servicios puede contribuir en dos niveles de intervención:

- Identificando necesidades de los clientes por medio de metodologías de Diseño Centrado en las Personas.

- Gestionando un nuevo enfoque para el diseño de propuestas de valor a través de herramientas de visualización. 


\section{DISEÑO DE SERVICIOS}

Diseñar un servicio es lograr un balance entre los intereses de los stakeholders principales (clientes, el negocio o la organización de ventas) y la operación u organización de suministro (Dhanesha et al., 2009). La perspectiva de los clientes debe ser el punto de partida del diseño de servicios. Grönroos (2000) plantea que la mayor contribución del diseño a la innovación en servicios es que todo proceso se puede plantear desde la óptica del usuario. Que los clientes participen en la fase de diseño del servicio asegura su éxito en términos de lealtad y satisfacción. (Yuan, 2008).

El diseño de servicios contribuye a la adquisición de la visión holística necesaria para considerar el contexto completo de entrega de la propuesta de valor servitizada, incluyendo todas las interacciones entre personas, artefactos físicos, espacios y tecnologías (Blomkvist, Holmlid, y Segelström, 2010). Esto es cada vez más importante cuando se piensa en las preocupaciones económicas y ambientales, es sabido que se necesita hacer más con menos (Hunter, 2015).

Asimismo, Provee de herramientas de visualización capaces de manejar la complejidad de las ofertas integradas de producto-servicio (Morelli, 2006). Ampliando esta información, el diseño de servicios se basa en herramientas metodológicas que permiten observar, analizar y entender la realidad sobre una situación concreta o necesidad del público objetivo. Conocer la relación de los usuarios con las soluciones propuestas antes de su implementación o producción, permite realizar una verificación sobre qué elementos hay que intervenir para mejorar y ser aceptados por el usuario, con el objetivo final de diseñar una experiencia predecible y estructurada.

Estos procesos de diseño, en su mayoría, se basan en el Design Thinking, que consiste en aplicar la mentalidad y las herramientas de los diseñadores a la resolución de problemas complejos. Hace referencia a la manera de pensar del diseñador que, a través de un razonamiento deductivo y poco convencional en el mundo empresarial, busca formular preguntas que deben ser contestadas a través de la comprensión del entorno. Es decir, la solución no deriva del problema, sino que encaja en él (Vianna, et al., 2016).

El Design thinking usa la sensibilidad y los métodos del diseñador para hacer coincidir las necesidades de las personas con lo que es tecnológicamente factible y con lo que una estrategia viable de negocios puede convertir en valor para el cliente y en una oportunidad de mercado (Brown, 2008; Harvard Bussines Review, 2008. pp 63-72). 
Al Realizar una revisión de los modelos basados en Design Thinking que se pueden aplicar al diseño de servicios, se han querido destacar los siguientes, que son los que muestran sutiles diferencias entre ellos:

- Modelo IDEO-d.school.

- Human Center Design.

- Doble diamante.

- Service Design Thinking.

- Diseño de servicios en 5 pasos.

Al igual que en la definición de servicios, el Service Design Thinking establece los puntos de contacto que se deben tener en cuenta para tangibilizar la experiencia del usuario:

- Personas: Asumen diferentes roles y actitudes al interactuar con otros actores en el servicio.

- Objetos: Facilitan y mejoran la experiencia y son la evidencia del servicio para el usuario.

- Canales: Deben ser claros y cercanos, transmiten información al usuario y facilitan los procesos del servicio.

- Procesos: Conjunto de operaciones lógicas que determinan el orden, la claridad y el entendimiento del servicio.

Manzini (2009) indica dos factores a considerar en el diseño de servicios:

- La transición hacia una sociedad en red y del conocimiento: La vinculación activa entre los diferentes agentes del sistema. El diseño ya no es solitario y unidireccional.

- Desarrollo sostenible, desde sus tres pilares: social, económico y medioambiental. 


\section{EMOCIONES E INCONSCIENTE}

La ingeniería Kansei nació como una tecnología ergonómica para el desarrollo de productos orientados a las necesidades y sentimientos del consumidor. Como indica Ruiz (2013) lo que fundamenta y construye fidelización es de carácter emocional y se basa en la confianza. Lo emocional es humano, dotar a la empresa de rasgos de personalidad y que ésta los transmita a su mercado ayudará a conseguir y fidelizar clientes.

Por lo tanto, la experiencia de usuario y su relación emocional con los servicios, son los que conseguirán una fidelización hacia la empresa. Para ello indica que el manual de experiencia corporativa debe tener en cuenta los siguientes puntos de interacción con el cliente, permitiendo mejorar su experiencia con el fin de fidelizarlo:

Target, Valores de la personalidad corporativa, Colores corporativos, Marca y aplicaciones de la misma, Odotipo y otros aromas corporativos, Música corporativa e hilo musical, Estancias corporativas, Internet, Personal, Marca personal y Gestión de errores.

Según Epstein (1994) las personas perciben la realidad de, al menos, dos maneras: cognitiva, que es considerada analítica y racional, y afectiva, que se entiende como intuitiva y experimental. Recientes estudios han determinado que hasta el 95\% de nuestra toma de decisiones se lleva a cabo bajo el umbral de la consciencia. Nuestros sentidos captan alrededor de 11 millones de bits de información cada segundo, de los cuales el cerebro consciente tan solo puede procesar, aproximadamente, 40 bits por segundo. El subconsciente o la manera afectiva de procesar la información es 200 veces más rápida. Esto es debido, principalmente, a que el procesamiento cognitivo conlleva una búsqueda automática y coincidente en la base de datos de la experiencia.

En cierto modo, es como si el cerebro buscase en su base de datos de la memoria eventos relacionados, incluyendo su valencia y sus consecuencias. Esto significa que las zonas del cerebro de la racionalidad no pueden funcionar aisladas de las zonas de regulación biológica-emocional. Los dos sistemas se comunican y afectan la conducta en forma conjunta, y consecuentemente, el comportamiento de las personas. 
Como expresa Braidot (2005), las encuestas, las entrevistas en profundidad y los focus groups sólo proporcionan, información superficial sobre las causas que verdaderamente subyacen en la conducta de compra y consumo. Esto se debe a que tanto las respuestas a un cuestionario como las conversaciones guiadas durante la investigación motivacional obtienen información basada únicamente en la reflexión consciente cuando en realidad la mayor parte de las decisiones que se toman tienen su origen en motivos no conscientes. La clave ya no está en analizar lo que dicen los clientes o en observar la forma en que se comportan, sino en indagar las causas que subyacen en su conducta. Cada vez que nuestro cerebro es sometido a estímulos a través de los sentidos (vista, oído, tacto...), su actividad y sus consecuencias desde el punto de vista psicofisiológico y conductual se revela en una serie de señales (eléctricas, magnéticas, químicas...). A partir de esto, se han desarrollado diversas herramientas tecnológicas, de funcionalidad neurológica y psicofisiológica, para medir e interpretar esta información emocional.

Actualmente las empresas, en vez de solicitar a los diseñadores que conviertan una idea ya desarrollada en otra más atractiva para los consumidores, están pidiendo que creen ideas que satisfagan mejor los deseos y las necesidades de los consumidores. El primer papel es táctico y genera una creación limitada de valor. El segundo es estratégico y conduce a enormes formas nuevas de valor (Brown, 2008).

\section{METODOLOGÍA}

Las investigaciones, tanto desde el punto de vista psicológico como neurocientífico, aportan nuevas aclaraciones del funcionamiento del cuerpo humano y especialmente del cerebro en relación a los estados emocionales y su relación con el entorno, a la vez que aportan nuevos interrogantes. Todo esto implica que la incorporación de dichos avances en el proceso de diseño sea una ardua tarea y se traduzca en una falta de consenso.

Uno de estos caminos es el planteado por Monestina (2012), en el que a través de una metodología de diseño convencional e implementando una serie de herramientas propias del diseño emocional junto con dispositivos de medición de parámetros psic ofisiológicos y conductuales, mide de forma fiable y sencilla las emociones de los usuarios haciendo que se vean reflejadas en el diseño del producto a desarrollar y buscando una alternativa a los procesos tradicionales. 
En el camino de validar el uso de los dispositivos neurocientíficos y aspectos emocionales dentro del proceso de diseño, se analizará el desarrollo de un "servicio de gestión y cartas virtuales de restaurantes". Para este estudio solamente se presentará el diseño y validación sobre un "momento de verdad" de la interacción cliente-servicio.

El proyecto se gestiona con la metodología del Design Thinking y desde la perspectiva del Human Center Design, debido a que esta considera al usuario como centro del proceso. También se aplicará de forma sencilla el Kansei tipo I para incorporar la parte emocional en el diseño de las alternativas que posteriormente se validarán mediante técnicas convencionales y neurocientíficas.

Este estudio fue experimental, La esencia de esta concepción de experimento es que requiere la manipulación intencional de una acción para analizar sus posibles resultados Creswell (2013a) y Reichardt (2004) llaman a los experimentos estudios de intervención, porque un investigador genera una situación para tratar de explicar cómo afecta a quienes participan en ella.

Para ello se selección de tipo exploratorio con control experimental de balanceo, Según Hinkelman. K. \& Kempthorne. O.(1994), citado por Mendoza(2016) estos estudios exploratorios son aquellos en el cual el investigador está interesado en encontrar los factores que tienen influencia sobre las ejecuciones de cierto proceso, Según Hinkelman. K. \& Kempthorne. 0. (1994), son aquellos en el cual se trata de comparar el "mejor" procedimiento encontrado en el experimento exploratorio con un procedimiento establecido o un producto. Se utilizó control experimental de balanceo, este control según Ramírez (2014) es útil cuando no es posible o conveniente en el experimento mantener las condiciones constantes, el experimentador debe recurrir a la técnica de equilibrar el efecto de las variables extrañas, entre estas la espera de turno para el uso de eye-tracking, el intercambio de comentarios entre participantes por no poder aislarlos completamente

Se utilizó una muestra de 30 sujetos (hombres y mujeres) entre 25 y 40 años. Las Instalaciones de experimentación fueron en empresa Emotional Factor Neurodesign, Oviedo España. Se desarrollaron dos pruebas distintas de validación, que permitieron comparar los resultados mediante técnicas convenciones y tecnológicas, dividiendo a los sujetos en ambas fases para evitar sesgos en la evaluación del primer impacto emocional en su uso, la aplicación de la 
entrevista de tipo estructurada de característica de preguntas abierta para lograr la identificación de características semánticas

\section{DESARROLLO}

Fases del Human Center Design:

\section{ESCUCHAR}

En esta fase se analiza a los usuarios potenciales, realizando un perfil de ellos mediante la herramienta Personas. Posteriormente se realizó una búsqueda de necesidades describiendo el proceso de pre-servicio, servicio y post-servicio a través de una StoryTelling (Narracion). Los problemas detectados se organizaron por afınidad, generando diferentes grupos: interacción pedido, interacción camarero, tiempos de espera, información de los productos y cobro.

A esta información se le añadió un análisis de competidores y tendencias del mercado, que permitió, finalmente, formar el campo de propiedades del producto. En esta fase se realizó la búsqueda de las palabras Kansei, que son aquellas emociones o sensaciones que se desea transmita la aplicación, llegando a seleccionar 10 palabras.

\section{CREAR}

En función del campo de propiedades y las palabras Kansei definidas en la fase anterior, se procedió a generar diferentes soluciones y prototipos de las soluciones. Tras realizar un filtro de las soluciones mediante diferentes técnicas, se seleccionaron 3 soluciones finales.

En este caso, los criterios de evaluación utilizados han sido los siguientes: Navegabilidad, simplicidad, claridad, cantidad de información, complejidad de información, intuición, desarrollo técnico (en función del tiempo y recursos disponibles para el primer prototipo), business intelligence (Capacidad de obtención de datos en relación a lo que ve o hacer el usuario) y seguridad.

\section{ENTREGAR}

En esta fase se prototiparon las soluciones y se desarrolló la validación de las mismas con potenciales usuarios. 
La primera fase se realizó mediante un diferencial semántico propuesto por la Ingeniería Kansei y una encuesta final (ver Figura 3).

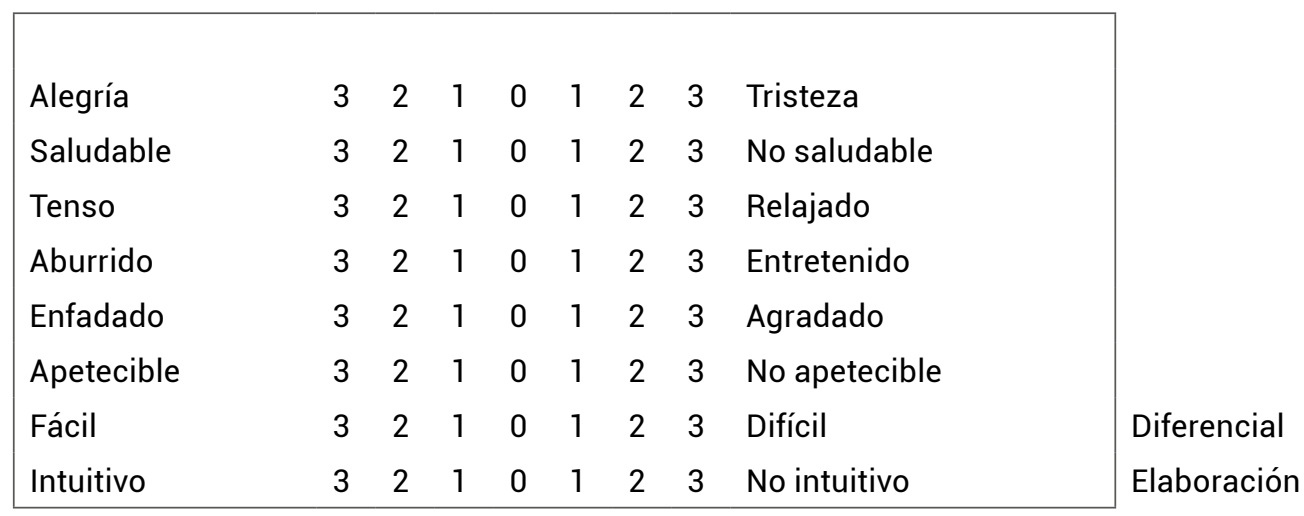

Figura 1. Diferencial semántico.

Fuente: Elaboración propia.

Para la entrevista se definieron las siguientes preguntas abiertas:

- ¿Cuál es la alternativa que más te ha gustado? ¿Por qué?

- ¿Qué es lo que más te ha llamado la atención?

- ¿Qué es lo que menos te ha gustado de lo que has visto?

- ¿Qué te ha parecido la prueba? ¿Cambiarías algo? ¿Qué te ha llamado la atención?

La segunda fase se desarrolló a través de equipos de análisis psicofisiológico y conductual: Software de reconocimiento emocional en la expresión facial, brazalete de actividad electrodérmica y el eye-tracker (ver Figura 4). 
Administración estratégica del Service-Dominant Logic en el Neurodiseño de un Servicio de Gestión y Cartas Virtuales de Restaurantes

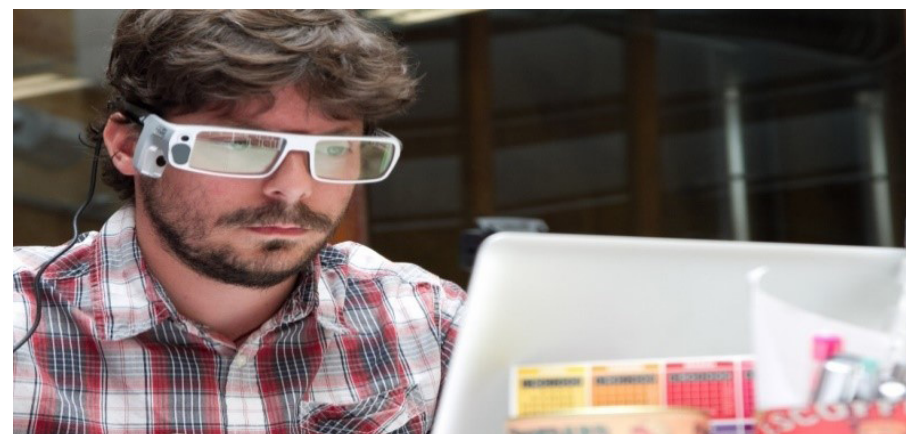

Figura 2. Usuario realizando prueba con el eye-tracking.

Fuente: elaboración propia

Ambas fases consistieron en la visualización estática de las diferentes alternativas de la aplicación. Cada imagen contaba con una tarea específica, por ejemplo, "buscar menú Carnes" (ver Figuras 3 y 4).
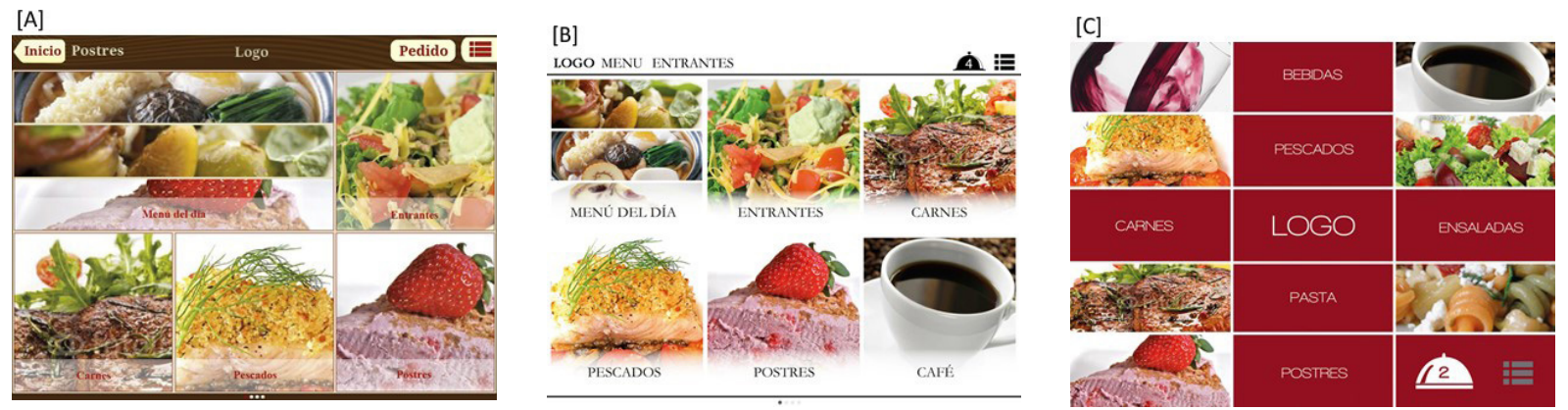

Figura 3. Página principal. 3A. Alternativa 1. 3B. Alternativa 2. 3C. Alternativa 3.

Fuente: elaboración propia

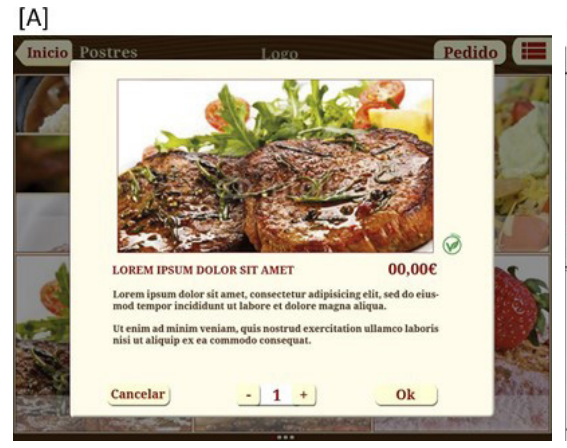

[B]

[C]

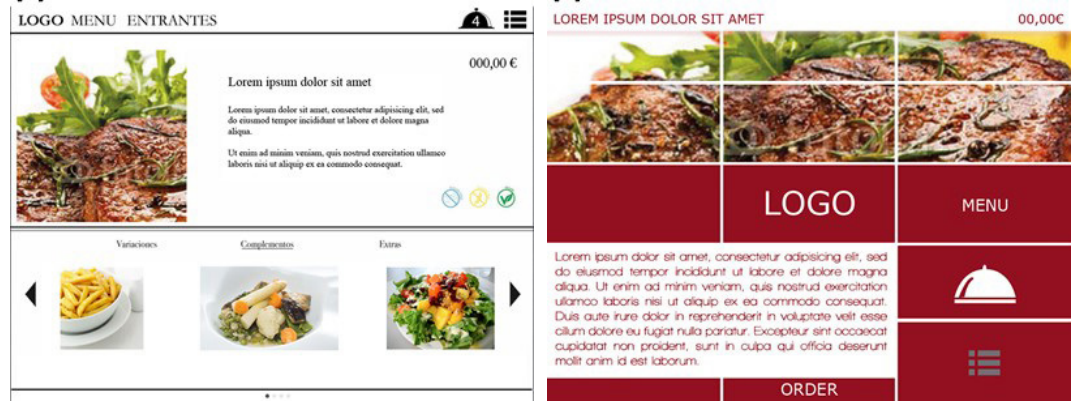

Figura 4. Página detalle. 4A. Alternativa 1. 4B. Alternativa 2. 4C. Alternativa 3.

Fuente: elaboración propia 


\section{RESULTADOS}

Tras analizar los resultados de los diferenciales semánticos de todos los usuarios ( 7 mujeres y 8 hombres) que han realizado la prueba, se han obtenido las Figuras 6 y 7 .

Las gráficas muestran el diferencial de puntuación entre cada par semántico. Si el resultado es positivo en la gráfica, significa que son las emociones o sensaciones de carácter positivo (alegría, saludable, relajado, entretenido, agradado, apetecible, fácil e intuitivo). Si, por lo contrario, el resultado es negativo, corresponden a emociones negativas (tristeza, no saludable, tenso, aburrido, enfadado, no apetecible, difícil, no intuitivo).

Lógicamente, la alternativa seleccionada será aquella que tenga mejores puntuaciones de carácter positivo.



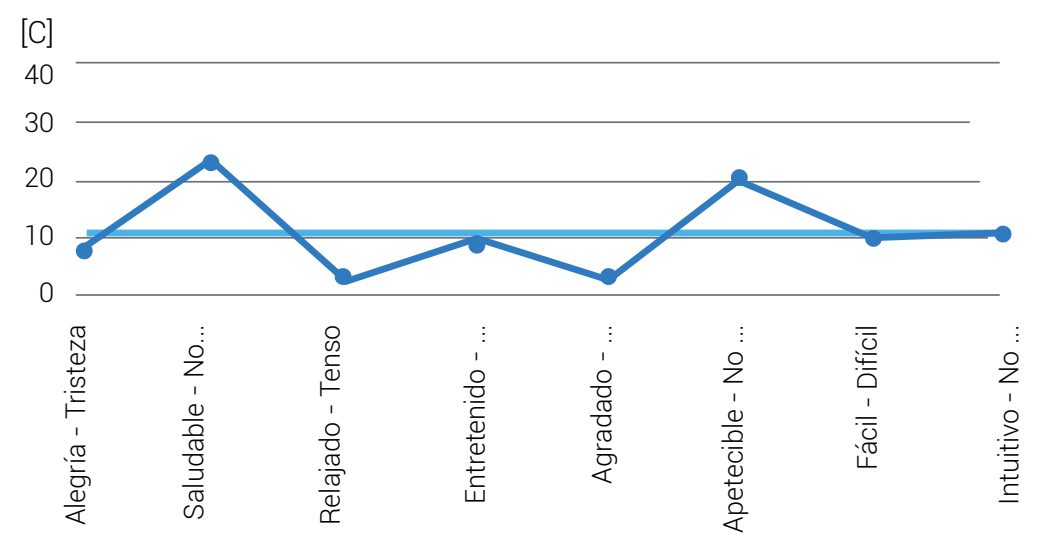

Figura 5. Resultados página principal. 5A. Alternativa 1. 5B. Alternativa 2. 5C. Alternativa 3.

Observando los resultados de la Figura 6, se ve claramente que la Alternativa (3) es la peor valorada en todos los aspectos, por lo que queda descartada. Las dos primeras alternativas son bastante similares en la puntuación de todos los diferenciales semánticos, siendo la Alternativa (2) la que transmite sensación de mayor facilidad respecto de la Alternativa 1. Por lo tanto, se puede considerar que, por los resultados obtenidos, la Alternativa 2 de las pantallas principales es la mejor valorada.

Si lo cotejamos con los datos obtenidos de la entrevista realizada al final de la prueba, no se aprecia esta similitud en los resultados entre las dos primeras alternativas, ya que 11 de los 15 encuestados destacan en la Alternativa 2 la interfaz intuitiva, la sencillez, la sutileza, la limpieza, el fondo blanco para descansar la vista y la cualidad de apetecible.

Comparando los resultados de la Figura 7 , se ve claramente que la página de detalle de la Alternativa 2 es la mejor valorada. Muestra una aproximación en ciertos diferenciales con la Alternativa 1 como, por ejemplo, en "intuitivo-no intuitivo". Asimismo, se considera más fácil, saludable, apetecible, transmite mayor relajación y más entretenimiento que la Alternativa 1. Por otro lado, la Alternativa 3 ha sido la única con valoración negativa. Al igual que en la pantalla principal, esta alternativa queda descartada. 
Al comparar estos resultados con lo descrito por los usuarios durante la entrevista, se asemeja más a los datos obtenidos de los diferenciales. 10 de los 15 usuarios han elegido en esta ocasión la alternativa 2 de la página de detalle, por las mismas razones descritas para la página principal: sencillez, limpieza, más intuitiva, etc.

La entrevista también ha indicado que, de forma general, la Alternativa 3 no es atractiva debido a su estructura y el color rojo dominante. Estas apreciaciones se observan en los diferenciales semánticos.
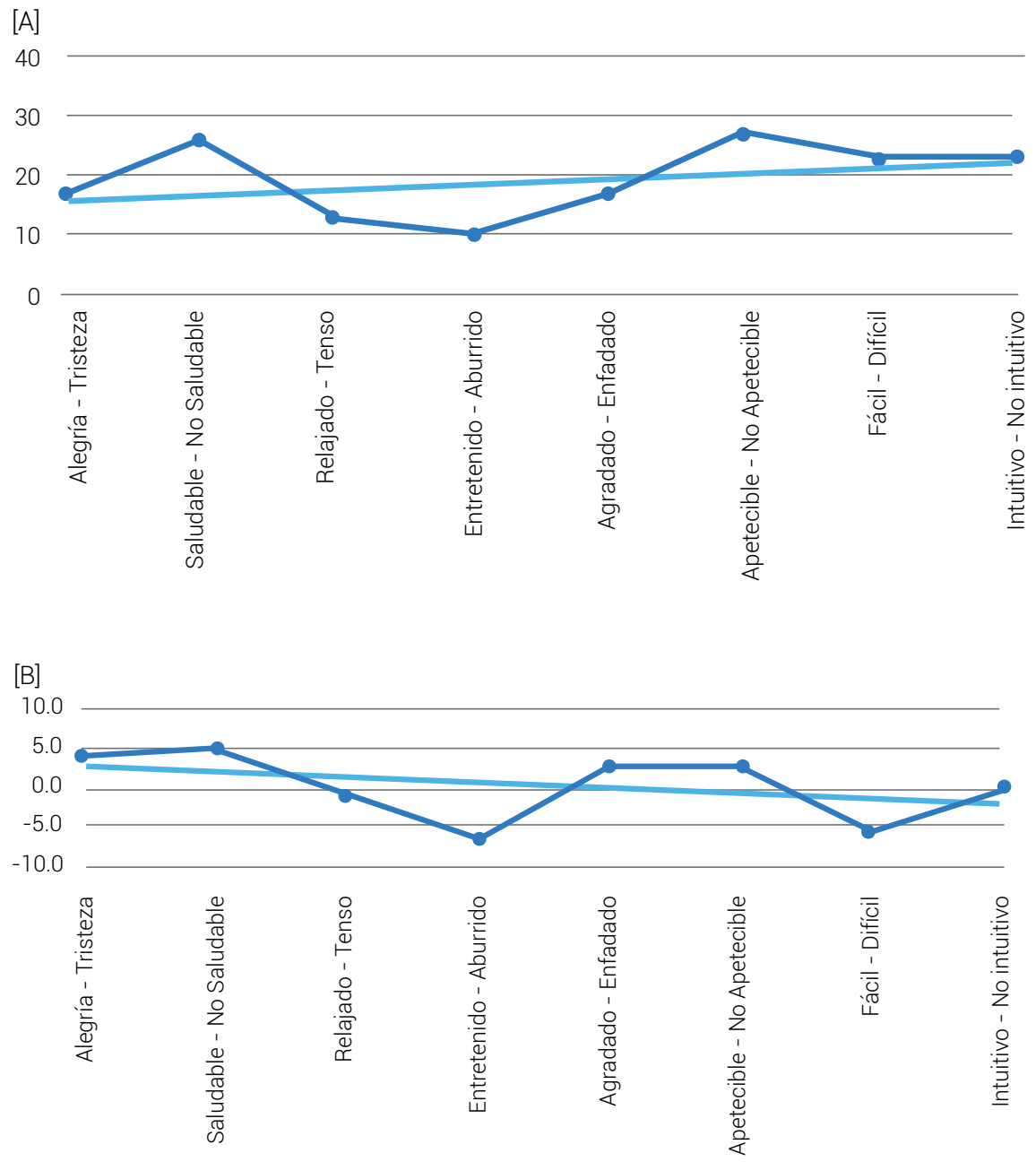


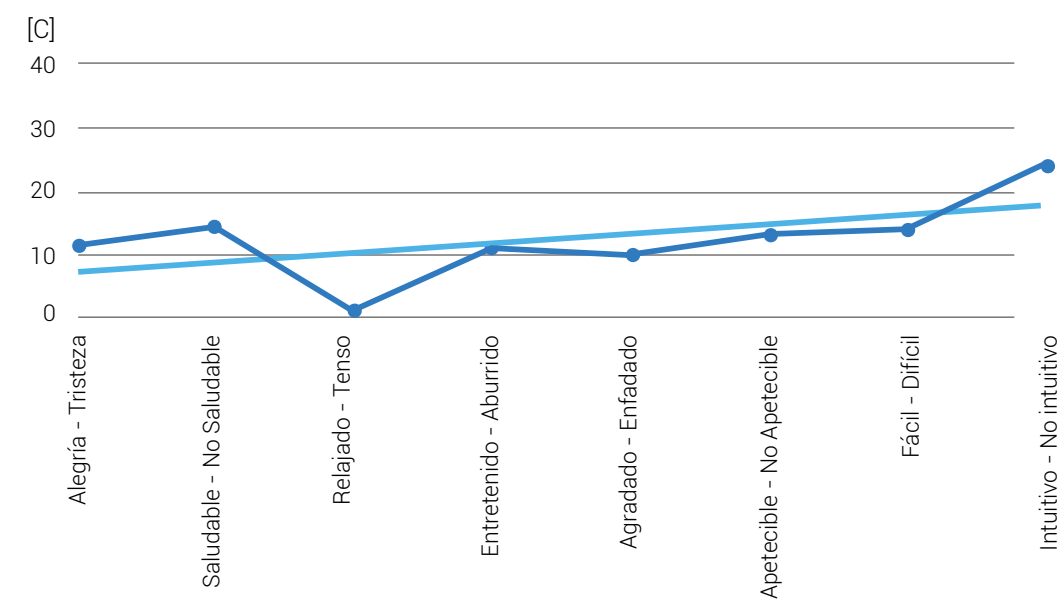

Figura 6. Resultados página detalle. 6A. Alternativa 1. 6B. Alternativa 2. 6C. Alternativa 3.

Fuente: elaboración propia

Finalmente, los usuarios encuestados han indicado que la prueba es interesante y correcta, pero han encontrado dificultades en el diferencial semántico, consideran que son difíciles de catalogar en ciertas circunstancias, que pueden verse influenciados por anteriores respuestas o confundirse al valorarlos rápidamente. De igual forma, en función de cada imagen o persona pueden tener diferentes significados.

Se puede considerar, desde un punto de vista emocional y según los datos arrojados por los diferenciales semánticos, que la Alternativa (2), tanto en la página principal como en la de detalle, es la preferida por los usuarios.

\section{RESULTADOS PRUEBA CON DISPOSITIVOS}

Esta prueba se ha realizado, al igual que el Kansei, a 7 mujeres y 8 hombres. La prueba está compuesta por 3 etapas:

1. Entrenamiento: Preparación de los sujetos para la interacción con las herramientas de medición.

2. Fase 1: Páginas principales de la aplicación.

3. Fase 2: Páginas de detalle de la aplicación. 
Inicialmente, el software de reconocimiento facial de las emociones ha identificado que las emociones más frecuentes son sorpresa, alegría y tristeza. Los datos de algunos sujetos se muestran extremos, por un lado, una altísima variabilidad en cuando a la frecuencia y tipos de emociones y, por otro lado, una reacción emocional neutral. Esto debido a la variabilidad que existe entre las personas tanto a nivel cognitivo como emocional.

Las emociones más sobresalientes se muestran en la mitad y final del tramo temporal del vídeo, es decir, una vez pasada la fase de entrenamiento comienzan las fases experimentales. Dichas emociones probablemente se deben al cambio en el espacio de los componentes mostrados en el vídeo. Con esto se hace referencia a que de ocho imágenes que muestra el vídeo, tres de ellas son exactamente iguales en cuanto a contenido se refiere, pero no a la distribución espacial de los componentes. Además, en estas imágenes el idioma que lo complementa es el castellano a diferencia de las últimas fotografías cuyo idioma es el latín, elemento que puede influir también en los sujetos, provocando rechazo en los mismos por ausencia de asociación entre éstas y las anteriores imágenes. Finalmente, la falta de asociación y/o explicación genera reacciones de extrañeza mostrándose emociones con valencia negativa.

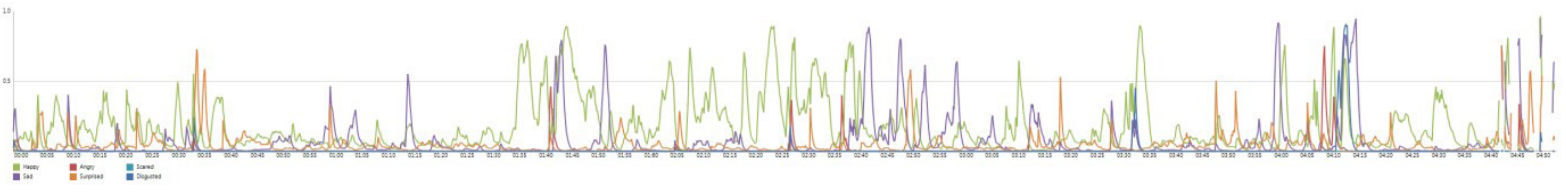

Figura 7. Tramo temporal-emocional. Fuente: elaboración propia

En lo que respecta a la valencia (positiva o negativa), se ha podido identificar que, según los datos obtenidos, la Fase 1 presenta resultados más positivos que la Fase 2. La media de la valencia es más positiva para la Fase 1 que para la Fase 2, pero a su vez, dentro de cada una se ha identificado cual es la diapositiva mejor valorada a nivel emocional.

Para la Fase 1, la diapositiva que mejor valor obtiene es la Alternativa 1, después la 2 y por último la 3. Para la Fase 2, la diapositiva que reúne mayor valencia positiva es la Alternativa 2, después la 3 y por último la Alternativa 1. 
La actividad electrodérmica y temperatura medida a través del brazalete es bastante constante en el tiempo, no se aprecian cambios significativos. Respecto a los datos obtenidos de la herramienta eye-tracker, se observa que la media del muestreo se centra en la parte media superior y en la parte izquierda inferior, según lo presentado por el heat map o mapa de calor. Estas zonas que muestran los lugares donde el usuario ha fijado por más tiempo la mirada es donde han aparecido los estímulos tanto en imágenes, como en palabras que representan las imágenes y el texto en latín.

La atención aumenta en estímulos-fotografía "carne", siendo uno con mayor duración, el estímulo-palabra LOGO, y algunos estímulos-textos en idioma latín. Es posible indicar que hay una asociación entre el reconocimiento facial de emociones y la atención prestada a los estímulos, ya que en la línea emocional-temporal se identifican los estímulos con mayor potencia informativa correspondientes a los identificados a través del seguimiento ocular.

Según los dispositivos, la mejor valorada es la Alternativa 2, seguido de la Alternativa 3, resultados diferentes de los representados mediante el diferencial semántico.
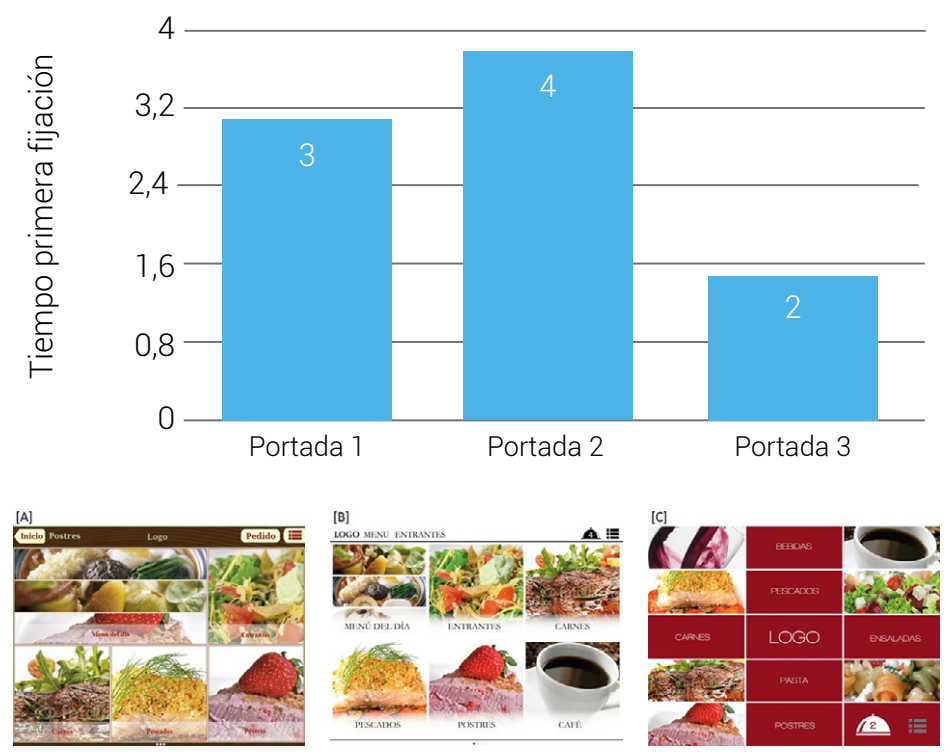

Figura 8. Tiempo de fijación en el "menú carnes" analizado con el eye-tracking para las 3 alternativas de páginas principales.

Fuente: elaboración propia 
Uno de los resultados más sorprendentes se obtuvo en el análisis a profundidad de la Alternativa 3. Según se puede apreciar en la Figura 9 hay una percepción de que su uso es difícil. Sin embargo, también se puede observar que refleja los resultados del análisis del eye-tracker, el tiempo hasta que se produce la primera fijación del área "menú carnes" es muy inferior al resto de alternativas. Es decir, desde un punto de vista consciente los sujetos valoran de forma negativa la usabilidad de la Alternativa 3, mientras que los datos del eye-tracker, que demuestra la parte inconsciente, arrojan que permite una mayor rapidez y facilidad de uso.

\section{CONCLUSIONES}

Corroborar que es posible realizar una selección de alternativas mediante criterios emocionales con la utilización de dispositivos de medición de parámetros conductuales y psicofisiológicos, aportando datos fiables y objetivos. De igual manera los participantes identifican que es muy compleja y subjetiva la selección de palabras Kansei, con muchas más horas de trabajo que la utilización de los dispositivos, debido Dificultad lingüística de los usuarios y del equipo de trabajo en la capacidad de transmisión, compresión y decisión de valoración en los diferenciales semánticos, y en la capacidad de comunicación en las entrevistas.

Diferencias entre los datos obtenidos de los dispositivos y las respuestas del usuario en el cuestionario, tras racionalizar la respuesta. La opción tres demostró ser menos funcional pero en términos de diseño e imagen, inconscientemente se determinó que es la que mayor atracción tiene para el usuario/participante

Medición precisa de los estados emocionales a lo largo del proceso de diseño mediante los dispositivos tecnológicos.

\section{REFERENCIAS}

Albrecht, K. (1992). Servicio al cliente interno: cómo solucionar la crisis de liderazgo en la gerencia intermedia. Barcelona: Paidós.

Aurich, J.; Mannweiler, C. \& Schweitzer, E. (2010). How to design and offer services successfully. Cirp Journal of Manufacturing Science and Technology. 2: 136-143. 10.1016/j.cirpj.2010.03.002. 
Baines, T.S. \& Lightfoot, H. (2013). Made to serve. How manufacturers can compete through servitization and product-service systems. Chichester, UK: Wiley.

Berdugo C., C.; Oviedo-Trespalacios, Ó.; Peñabaena N., R.; Luna-Amaya, C. \& Nieto-Bernal, W. (2014). Diseño y desarrollo de servicios: una nueva perspectiva desde el ciclo de vida. Interciencia, 39(2): 111-115. Febrero, 2014.

Bhamra, T.; Moultrie, J. \& Thurston. P. (2014). Service design and manufacturing. En: D. Sangiorgi, A. Prendiville, \& A. Ricketts (eds.). Mapping and developing service design research in the UK. http:// www.servicedesignresearch.com/uk/wp-content/uploads/2014/06/Mapping-and-DevlopingSDR-in-the-UK.pdf.

Bitner, M.J. \& Brown, S.W. (2008). The service imperative. Business Horizons, 51(1): 39-48.

Blomkvist, J.; Holmlid, S. \& Segelström, F. (2010). This is service design research: yesterday, today and tomorrow. En M. Stickdorn \& J. Schneider (eds.). This is service design thinking. Amsterdam: BIS Publishers. pp. 308-315.

Dhanesha, K.A.; Hartman, A. \& Jain, A.N. (2009). A model for designing generic services. IEEE Int. Conf. On Services Computing, SCC'09.

Duque, E.J. (2005). Revisión del concepto de calidad del servicio y sus modelos de medición. Editorial Innovar. Enero-junio 2005. pp. 64-80.

Fisher, L. \& Navarro, V. (1994). Introducción a la investigación de mercado (3a ed.). México: McGraw-Hill Interamericana S.A. de C.V.

Galindo M., M.A. (2009). La importancia del sector servicios en el ámbito industrial. Econ. Industr. 374: 15-20.

Grönroos, C. (2000). Relationship marketing: interaction, dialogue and value. Revista Europea de Dirección y Economía de la Empresa, 9(3): 13-24. 
Hara, T.; Arai, T. \& Shimomura, Y. (2006). A concept of service engineering: a modeling method and a tool for service design. Paper presented at the Int. Conf. on Service Systems and Service Management.

Hunter, M. (2015). What is service Design? http://www.designcouncil.org.uk/.

Iriarte, I.; Justel, D.; Alberdi, A.; Val, E. \& González, I. (2016). Diseño de servicios para la servitización. Experiencias con empresas manufactureras vascas a través de la colaboración universidadempresa. Universia Business Review, (49): 146-181.

Kamp, B. (2016). La servitización como estrategia para la evolución competitiva de la industrial. Economistas, (150): 76-84.

Kamp, B.; Ochoa, A. \& Díaz, J. (2016). Smart servitization within the context of industrial user-supplier relationships: contingencies according to a machine tool manufacturer. International Journal of Interactive Design and Manufacturing, 10.1007/s12008-016-0345-0, 2016.

Kotler, P. (1997). Mercadotecnia. México: Prentice-Hall.

Mayer, K.J., Bowen, J.T. \& Moulton, M.R. (2003). A proposed model of the descriptors of service process. Journal of Services Marketing, 17(6): 621-639.

Mendoza, H. (29 de 04 de 2016). Diseño Experimental. http://red.unal.edu.co/cursos/ciencias/2000352/ index.html

Morelli, N. (2006). Developing new product service systems (PSS): methodologies and operational tools. Journal of Cleaner Production, 14(17): 1495-1501.

Neely, A. (2013). What is servitization? Accessed on 20 June 2016. http://andyneely.blogspot.com. es/2013/11/what-is-servitization.html.

Porter, M.E. (1979). How competitive forces shape strategy. Harvard Business Review, 57: 86-93. March-April. 
Ramírez, E. (10 de 09 de 2014). Departamento de Psicologia. http://www4.ujaen.es/ eramirez/ Descargas/tema6

Tung, W.F. \& Yuan, S.T. (2008). A service design framework for value co-production: insight from mutualism perspective. Kybernetes 37: 226-240.

Vladimirova, D.K. (2012). Transformation of traditional manufactures towards servitized organizations. PhD Thesis. Cranfield, UK: Cranfield University. 